\title{
Postoperative bacteremia is associated with early vascular complications in pediatric liver transplant recipients with biliary atresia
}

Kyong Ihn ${ }^{1}$, Ji-Man Kang ${ }^{2}$, Eun Jin Kim¹, Juhan Lee ${ }^{1}$, Jae Geun Lee ${ }^{1}$, Dong Jin Joo ${ }^{1}$, Soon II Kim ${ }^{1}$, Myoung Soo Kim

${ }^{1}$ Division of Transplantation, Department of Surgery, Severance Hospital, Seoul, Korea
${ }^{2}$ Division of Infection, Department of Pediatrics, Severance Hospital, Seoul, Korea

Background: Bacteremia after liver transplantation (LT) frequently occurs and is a potentially severe complication affecting patient and graft survival. Children with biliary atresia (BA) have an increased risk of recurrent cholangitis and heightened risk of clinically significant infections. This study evaluated the impact of bacteremia after pediatric LTs on clinical outcomes.

Methods: After the exclusion of retransplantation, a total of 63 patients with BA less than 18 years-old were performed LT between April 2006 and September 2019. The patients were divided into two groups, according to the occurrence of post-LT bacteremia within 1 month (bacteremia vs. no bacteremia: 14 [22.2\%] vs. 49 [77.8\%]).

Results: Compared with BA patients with no post-LT bacteremia, BA patients with bacteremia were significantly younger at the time of $L T$ (1.67 years vs. 3.71 years, $P=0.024)$, and had higher preoperative serum total bilirubin $(12.6 \mathrm{mg} / \mathrm{dL}$ vs. $7.6 \mathrm{mg} / \mathrm{dL}$, $\mathrm{P}=0.007$ ), higher GRWR (graft-versus-recipient weight ratio; 3.25 vs. $2.37, \mathrm{P}=0.020$ ), higher frequency of pre-LT bacteremia within 1 month $(28.6 \%$ vs. $4.1 \%, P=0.019)$. Three-year overall survival rates were similar ( $85 . \%$ vs. $79.1 \%, P=0.688)$, but vascular complications and reoperations were significantly frequent in BA patients with bacteremia $(57.1 \%$ vs. $26.5 \%, P=0.032 ; 42.9 \%$ vs. $4.1 \%$, $\mathrm{P}=0.001$, respectively).

Conclusions: The occurrence of bacteremia after pediatric LT was associated with increased numbers of vascular complications and reoperations. Although challenging, proper control of bacterial infections and early LT before developing uncontrolled cholangitis may be useful in reducing vascular complications and unexpected reoperations for patients with BA.

Corresponding author: Myoung Soo Kim

E-mail: ysms91@yuhs.ac

(c) The Korean Society for Transplantation

This is an Open Access article distributed under the terms of the Creative Commons Attribution Non-Commercial License (http://creativecommons.org/licenses/by-nc/4.0/) which permits unrestricted non-commercial use, distribution, and reproduction in any medium, provided the original work is properly cited. 